\title{
Eine unbekannt gebliebene Schrift Philipp Johann von Strahlenbergs
}

\author{
Michael Knüppel \\ Göttingen
}

\begin{abstract}
The following article deals with the widely unknown description of the „Bouchary”, which was published anonymously in 1723. As the writer shows, the work that is entitled "L'etat present de la Boucharie" goes back to the well-known Swedish-German officer, historian, geographer and Siberia-traveller Philipp Johann von Strahlenberg, who is known until now as the author of the famous description of Siberia and Central Asia entitled „Das Nord- und Ostliche Theil von Europa und Asia”, as well as a preliminary report („Vorbericht”) to this work and a lost edition of the known genealogical history written by the ruler of Qoqand, Abū-'1-Ġāzĩ Bahādur Hāan. After a general introduction of the text, the work on the "Bouchary" is presented in detail.
\end{abstract}

Keywords: Ph. J. v. Strahlenberg, description of Central Asia, history of the Kalmucks, Kalmuck wars, authorship of Ph. J. v. Strahlenberg, "Bouchary".

Es mag durchaus kein Mangel an Publikationen zu Leben und Schaffen Ph. J. v. Strahlenbergs $\left(1677^{1}-1747\right)$ bestehen. ${ }^{2}$ In einer Vielzahl von

1 Philipp Johann von Strahlenberg wurde nicht, wie häufig angegeben, 1676 (Henze, Dietmar: Strahlenberg (eigentl. Tabbert), Philipp Johann von. In: Enzyklopädie der 
Darstellungen zur ural-altaischen Theorie, ebenso wie in forschungsgeschichtlichen Beiträgen und biographischen Skizzen scheinen Leben und Werk des Geographen (resp. Kartographen), GelegenheitsHistorikers und -Ethnographen erschöpfend behandelt und seine Leistungen auf verschiedenen Gebieten dem Laien wie den Vertretern der von den Forschungen des ungewöhnlichen Gelehrten berührten Bereichen ebenso bekannt, wie die nicht wenigen Schwächen seines 1730 erschienenen Hauptwerkes „Das Nord- und Ostliche Theil von Europa und Asia ...". Und dennoch hält v. Strahlenbergs Leben, wie auch sein Werk selbst, noch nach rund drei Jahrhunderten so manche Überraschung für die angesprochenen Vertreter der verschiedensten

Entdecker und Erforscher der Erde. 25. Lieferung: Stoddart-Vadillo. Graz 2003, coll. 249-250; Jarring, Gunnar: Strahlenberg in Schwedischer Literatur und Wissenschaft. Eine bio-bibliographische Übersicht. In: UAJb, 48. 1976, pp. 121123; Krueger, John R.: The Kalmyk-Mongolian Vocabulary in Stralenberg's Geography of 1730. Asiatica Suecana, Early 18th Century, Documents and Studies, 1. Stockholm 1975; Sertkaya, Osman Fikri: Doğumunun 311 ölümünün 240. yıl sönümünde Philipp Johann von Tabbert-Strahlenberg (1676-1747). In: TK, 285. Januar 1987, pp. 16-27) geboren, sondern erst 1677 (wie vom Vf. an anderer Stelle bereits ausgeführt). Den Kirchenbüchern der Stadt Stralsund ist zweifelsfrei zu entnehmen, daß Philipp Johann Tabbert/ Strahlenberg am 6. Oktober 1677 in der St. Nicolai-Gemeinde getauft wurde (Kirchenbuch St.-Nicolai, p. 300 b; freundliche Mitteilung von Herrn Dr. Hacker [Stadtarchiv Stralsund]). Das Geburtsdatum ist hier nicht eingetragen, dürfte allerdings nur wenige Tage davor anzusetzen sein (im Falle der meisten Geburten/ Taufen aus dieser Zeit in der betreffenden Gemeinde liegen nur drei bis fünf Tage zwischen der Geburt und der Taufe).

2 Henze (2003), coll. 249-250; Jarring (1976), pp. 121-123; Krueger (1975); Sertkaya (1987), pp. 16-27. Vgl. daneben Bratt, Einar: Karolinen Philipp Johann von Strahlenberg och hans sibirka karta. In: Föreningen Armémusei vänner, Meddelande XXVII frän Kungl. Armémuseum. Stockholm 1966, 73-92; Novljanskaja, Marija Grigor'evna: Filipp Iogann Stralenberg, Jego raboty po issledovaniju Sibiri. Moskva, Leningrad 1966 (schwed. Übers. [v. Apazidis, Anita]: Novljanskaja, M. G.: Philipp Johan von Strahlenbergsforskningsarbeten om Siberien. Karlstad 1968); Strindberg, August: Philip Johan von Strahlenberg, hans karta och beskrivning över Asien. In: Antropologisk-geografiska sällskapets tidskrift, I. 1879 (Neudruck in: Kulturhistoriska Studier. Stockholm 1881. 18-27; vgl. die von John Landquist herausgegebene 55-bändige Werkausgabe Strindbergs [Samlade skrifter. 1911-1921], hier Bd. 4, pp. 28-41); Yarosh, G.: F. I. TabbertStralenberg - sputnik issledovatelya Sibiri D. G. Messerschmidta. In: Izvestiya Sibirskogo otdeleniya AN SSR. Seriya ob̌̌čestvennykh nauk. 1968 (I), pp. 68-72. 
Disziplinen bereit. So wurde dem Verfasser dieser Miszelle vor dem gemeinsam mit $M$. Ölmez besorgten Neudruck der englischen Übersetzung des Hauptwerkes v. Strahlenbergs, ${ }^{3}$ zu welchem ersterer einen Überblick über Leben und Werk des schwedischen Offiziers und Sibirienreisenden beigesteuert hatte, unterbreitet, daß sich hier wohl kaum Neues wird finden lassen und nachdem die Kollegen eines Besseren belehrt worden waren, von einigen in dem dann folgenden voreiligen Lob festgestellt, daß nun alles vorbildlich aufbereitet sei. Tatsächlich jedoch sind Leben und Schaffen v. Strahlenbergs noch immer weit davon entfernt, „erschöpfend“ behandelt zu sein - sofern so etwas wie eine erschöpfende Behandlung denn überhaupt denkbar ist. So soll denn auch der vorliegende kleine Beitrag nur verdeutlichen, wie viel hier noch $\mathrm{zu}$ tun ist und welch ausgedehnte Arbeiten noch der Erledigung harren.

In der zu v. Strahlenberg bestehenden Literatur wird, von nur wenigen Ausnahmen abgesehen, angenommen, daß selbiger nur drei Arbeiten verfaßt habe: neben dem bereits erwähnten Hauptwerk „Das Nord- und Ostliche Theil von Europa und Asia ...", 4 zum einen eine erste genaue Karte Zentralasiens und Nordeurasiens ${ }^{5}$ und zum anderen eine

3 Z. Zt. im Druck.

4 Strahlenberg, Philipp Johann v.: Das Nord- und Ostliche Theil von Europa und Asia: in so weit solches Das Gantze Russische Reich mit Sibirien und der grossen Tartarey in sich begreiffet. In einer Historisch-Geographischen Beschreibung der alten und neuern Zeiten, und vielen andern unbekannten Nachrichten vorgestellet, Nebst einer noch niemahls ans Licht gegebenen Tabula Polyglotta von zwey und dreyssigerley Arten tartarischer Völcker Sprachen und einem Kalmuckischen Vocabulario, Sonderlich aber Einer grossen richtigen Land-Charte von den benannten Ländern und andern verschiedenen Kupfferstichen, so die AsiatischScythische Antiqvität betreffen; Bey Gelegenheit der Schwedischen KriegsGefangenschaft in Russland, aus eigener sorgfältigen Erkundigung, auf denen verstatteten weiten Reisen zusammen gebracht und ausgefertigt. Stockholm (in Verlegung des Autoris) 1730 (Neudruck: With an Introduction by J. R. Krueger. Ed. Judit Papp. Szeged 1975 [SUA, 8]).

5 [Strahlenberg, Philipp-Johann v./ Matern, T. A./ Frisch, P. F.]: Nova descriptio geographica Tattariae magnae: tam orientalis quam occidentalis in particularibus et generalibus Territoriis una cum Delineatione totius Imperii Russici imprimis Sibiriae accurate ostensa; Serenissimo ac Potentissimo Regi Suecorum Gotorum et Vandalorum etc. etc. etc. Friderico Primo Domino suo Clementissimo Mappam hanc Geographicam quae Divina sic dispensante Providentia Originem suam Occasione Captivitatis Sibiricae traxit devotissimo 
mit ausführlichen Kommentaren versehene Übersetzung der „Geschichte der Mongolen und Tataren“ des Herrschers von Qoqand Abū-'1-Ġāzī Bahādur Hān, die heute bis auf einer auf v. Strahlenbergs Arbeit beruhenden französischen Übersetzung des Werkes, ${ }^{6}$ als verloren gilt. Daß v. Strahlenberg zudem einen „Vorbericht eines zum Druck verfertigten Werckes von der grossen Tartarey und dem Königreiche Sibirien" verfaßt hat, ${ }^{8}$ war offenkundig den wenigsten Autoren, die sich zu Leben und Schaffen des Sibirienreisenden geäußert haben, bekannt und noch wenigere haben diesen auch gelesen. Das mag vor allem in dem Umstand der äußerst geringen Verbreitung dieses „Vorberichts“ begründet liegen. Einem Mißstand, dem nun durch einen Neudruck dieser Schrift im Anhang zu dem oben erwähnten Reprint (der englischen Fassung) des Hauptwerkes abgeholfen wurde. Bei gründlicher Lektüre dieses „Vorberichts“ jedoch trifft der Leser auf den folgenden Hinweis v. Strahlenbergs in dem auf eine weitere, bislang unbekannt gebliebene Schrift des Sibirienreisenden verwiesen wird: ${ }^{9}$

Corde et obsequiosissima Mente offert, dicat, dedicatque humillimus ac obedientissimus Servus et Subditus P. I. v. Strahlenberg. J. A. Matern. P. J. Frisch sculpsit. Paris 1725.

6 [Aboulgasi Bagadur Chan] L'histoire genealogique des Tatars traduit du Manuscipt tatare d'Abulgasi-Bayadur-Chan, et enrichie d'un grand nombre de remarques authentiques ... Sur le veritable estat present de l'Asie sptentrionale, avec les cartes geographiques necessaires. Leyde 1726.

7 Das Werk, das zugleich den zweiten Teil seiner Arbeit „Das Nord- und Ostliche Theil von Europa und Asia ..." bildete - und im Anhang eines Briefes an den schwedischen Gesandten in den Niederlanden, Baron J. F. Preis ausführlich behandelt wird (Jarring, Gunnar: Das Schicksal der zentralasiatischen wissenschaftlichen Erbschaft von Philipp Johann Strahlenberg. In: CAJ 21. 1977, pp. 224-228, hier bes. 225-226) -, bestand aus einer erweiterten Neubearbeitung der Geschichte des Abū-'I-Ḡāzī Bahādur Hुān, einem „Traktat“ über Oguz Hūn, einer Beschreibung der „kleinen Bucharey“ (Ost-Turkistan), des „Königreiches Tanghut" (Tibet) und des "Reichs Boutan" (Bhutan), einem Bericht über eine Reise zu den westlichen Kalmücken, der Beschreibung einer russischen Expedition entlang des Irtyschs, einem Wegweiser für Reisen in Rußland, Sibirien und Zentralasien sowie zahlreichen Kupferstichen von Trachten und Kleidungen der Völker Sibiriens und der angrenzenden Regionen (ibid.).

8 Strahlenberg, Philipp Johann v.: Vorbericht eines zum Druck verfertigten Werckes von der grossen Tartarey und dem Königreiche Sibirien; mit einem Anhang von Gross-Russland, worinnen von dem Autore die Einrichtung und vornehmsten Contenta desselben, vorgetragen werden. Stockholm 1726.

9 Strahlenberg (1726), p. 47. 
„Denn es ist fast kein Völcklein darinnen, so mir nicht einiger massen nach denen einheimischen, alten und frembden Nahmen bekant seyn solte, fristet mit Gott das Leben und die Gesundheit, werde künfftig hinauch solche $\mathrm{zu}$ verbessern bedacht seyn, wie imgleichen das ans Licht erschienene Tractätlein l'etat present de la Bucharie à Cologne 1722 welches mich erinnere, einem gewissen Officirer in der Gefangenschafft negligemment aus dem Kopffe dictiret zu haben, so er mit einem kleinen Zusatz von den Lebens Manieren der Mahomedischen Bucharen embelliret hat" . Daß diese 47 pp. umfassende Darstellung der „Bucharei“ zu Beginn des 18. Jahrhunderts ${ }^{10}$ der Strahlenbergforschung bislang gänzlich unbekannt geblieben ist, erklärt sich wohl - abgesehen davon, daß sich in dem anonym erschienenen „Tractat“ selbst kein Hinweis auf v. Strahlenberg findet - vor allem aus dem oben angesprochenen Umstand, der sehr geringen Verbreitung des „Vorberichts“. Den Verfassern der wenigen Arbeiten, in denen dieser dennoch erwähnt wird, hat er vermutlich zumeist gar nicht vorgelegen, so daß auch die Erwähnung von „L'etat present de la Boucharie“" unbemerkt bleiben konnte. Hinzu tritt freilich wohl noch die Tatsache, daß diese Schrift, die übrigens 1723 erschien und nicht 1722, wie bei v. Strahlenberg irrtümlich angegeben, eine noch geringere Verbreitung erfuhr, als der „Vorbericht". Durch den glücklichen Umstand, daß beide Schriften sich in den Beständen der Staats- und Universitätsbibliothek Göttingen finden (Sign. 8 H RUSS 442/9 [,Vorbericht ..."] und 8 H AS II, 7520 [,L'etat present ..."]), waren die Identifizierung und die nachstehende Wiedergabe des Textes und der Abbildungen aus „L'etat present de la Boucharie" möglich. Der Verfasser hofft, hiermit $\mathrm{zu}$ weiteren Forschungen zu Ph. J. v. Strahlenberg anzuregen - und wer weiß schon, ob nicht doch eines Tages noch v. Strahlenbergs Bearbeitung der „Geschichte der Mongolen und Tataren“ wieder aufgefunden wird.

10 L'etat present de la Boucharie contenant vne description exacte de la situation, religion des moeurs, de la forme du gouvernement, et du commerce, avec une relation de la derniere revolution de ce pais la, de la fin tragique du prince BostoCham et de la vie de son successeur Contaisch Areptan prince aujourdhui. regnant, tire du manuscript d'un voyageur à Cologne 1723 . 
$[1]^{11}$

Chap. I.

De La Boucharie en general.

(1)

La Boucharie est un pays d'une etendüe considerable, sitüé entre celui de Turchestan, la grande Calmuquie, La Mer Caspienne, La Perse, Les Indes, \& la Mongalie.

(2) Les Geographes regardent ordinairement la Boucharie, comme faisant partie de la grande Tartarie. En effêt les Tartares, ou, pour mieux dire, Les Calmuques, en ont envahi une partie, mais leur domination n'empêche [2] pas, que la Boucharie, á l'exemple de la Chine, que les Tartares ont pareillement subjugée, ne doive être considerée come un Estât separé.

(3) La Boucharie est divisée en deux parties, la Grande \& la Petite.

C'est la derniere qui fera le principal objêt de la presente relation, j'en puis parler avec d'autant plus d'assurance, que j'ai eté moymême sur les lieux. Il est juste cependant, que je rapporte auparavant ce qui m'est connu de la premiere, c'est à dire, de la Grande Boucharie.

Chap. II.

De La Grande Boucharie.

\section{(1)}

La Grande Boucharie est situé sous le $36 . \&$ 45, degré, Elle confine, du coté du Nord, au pays de Turchestan \& à une partie de la Calmuquie Orientale, vers 1'occident à la Perse \& à la Mer Caspienne, vers le sud, aux Indes, [3] \& vers 1'orient, à la Petite Boucharie, dont elle est separée par de hautes montagnes qu'on apelle Parapomisus. ${ }^{12}$

(2) Elle étoit connuë, du téms D’Alexandre le Grand, sous le nom de Sogdiana, D'autres 1'appellent aujour d'hui Mauranneher, comme qui diroit, selon la langue du pays, Au dela des eaux, ou vers le coulant des

11 In den eckigen Klammern ist hier die Paginierung der Vorlage gegeben.

12 Die geographischen Angaben zur Ausdehnung der "Großen Bucharei" variieren in der Literatur des 18. Jahrhundert ganz erheblich, sowohl was die Ausdehnung in nördlicher Richtung betrifft (bis in Gebiete des heutigen Kasachstans hinein [häufig wird die "Große Bucharei“ als Süden der „Tartatarei“ bezeichnet]), als auch in Bezug auf die Ausdehnung nach Süden (noch in den Lexika des 19. Jahrhunderts werden hier Gebiete Afghanistans einbezogen). 
eaux, Mais dans la plus part des cartes Geographiques elle est appellée Terra Usbekorum, le pays des Usbeks, parce que les Usbeks avoient une fois subjugué les Bouchars, en s'emparant de leur Capitale, nommée Bouchara \& en se rendant presque tout le reste du pays tributaire.

(3) Les Usbeks continuënt même aujourd'hui, à les incommoder par leurs courses continuelles \& les obligent souvent à payer le tribut.

Et quoy que les Bouchars n'oublient rien pour se garantir de ce joug, ils n'en ont jamais pû venir entierement â bout, à l'exemple de bien d'autres Republiques, ils ont le malheur de n' [4] estre jamais d'accord entré eux mêmes au lieu d'unir leurs forces pour repousser l'ennemi commun, il y en a presque toujours parmi eux, qu'i l'assistent contre leur propres compatriotes. Tant il est Vrai, que les des unions domestiques, sont ordinairement fatales aux états qui y sont sujets.

(4) Il y a plusieurs Villes dans la Grande Boucharie, \& elles ont chacune son Cham, ${ }^{13}$ ou Regent les principales sont.

Bouchara, qu'on dit étre deux fois plus grand, que celle de Moscou en Russie, \& Balik, qui n'est pas moins considerable.

\section{Chap. III.}

De La Petite Boucharie. ${ }^{14}$

(1)

La petite Boucharie est separée de la Grande Boucharie, comme il a été dit ci-dessus, par le Parapomisus. [5] Elle est appellée par quelques uns la Mogulie, \& par d'autres Tzagatay, en memoire de Tzagatay, second fils de Zingis-Cham. ${ }^{15}$ Elle est située entre le 36. \& 42-me degré ayant pour voisins à l'orient, la Mongalie \& les deserts de la Chine; au sud, ceux des Indes, à l'occident, la grande Boucharie \& la Perse, \& au Nord, une Partie de la Mongalie, \& de la Calmuquie Orientale.

(2) L'etenduë du pays est de 200 . lieuës ou environ en longueur. Il y à plusieurs deserts, le reste consiste dans une vingtaine de Villes, qui ont chacune grand nombre de villages qui en de pendent.

13 Dies $=$ Hुān.

14 Eine Beschreibung der „kleinen Bucharei“ (= die Ost-Turkistan) sollte gemäß der von G. Jarring wiedergegebenen Anzeige ja auch v. Strahlenbergs zweites großes Werk beinhalten (s. o.).

15 Vgl, hierzu die nahezu identische Schreibung des Titels Čingis $\forall a ̈ a n$ in v. Strahlenberg (1730), pp. 12 und 45. 
Il n'y a que deux Villes, qui soyent de quelque consideration, savoir Jerken, qui est la Capitale du pays, fort grande \& bien peuplée, \& Cascar, située au pied des montagnes sus dites.

(3) Ce fût l'an 1683. que les Calmuques, sous leur Bosto-Cham, ou Bosugto-Cham occuperent la petite Boucharie. Apres la mort de BostoCham, ${ }^{16}$ [6] Zigan Araptam, ${ }^{17}$ son Neveu, en devint le Grand Contaisch, C'est le nom, que le peuple donne à ses Souverains. Je racconterai cidessous les particularités de cét evenement. ${ }^{18}$

(4) Zigan Araptan etant parvenû à la regence, ile établit l'ordre suivant dans la Petite Boucharie. Il instituä divers Magistrats subalternes, qui subsistent encore, \& qui sont subordonnez les uns aux autres les moindres gouvernent, chacun 10. Maisons ou familles, les seconds 100 . les troisiemes, 1000. \& tous dependent d'un Commandant General, que le Grand Contaisch, comme Souverain du pays, choisit ordinairement d'entre la race des anciens Princes des Bouchars.

Ces Magistrats jugent des differents qui naissent entre les sujets, \& sont dans l'obligation de faire rapport de tout ce qui se passe, chacun à son superieur, Par cette forme de gouvernement le Grand Contaisch tient un fort bon ordre dans son pays, oû tous [7] les habitants vivent en paix, $\&$ dans une parfaite union.

(5) Les Bouchars ne sont guerre belliqueux. Les armes dont ils se servent communement sont la lance, \& 1'arc, Il y en a cependant, qui ont des fusils $\&$ des arquebuses rayées, $\&$ les plus riches portent des jacques de maille.

(6) Pour juger de la force de ce peuple, on peut compter, que, quand

16 Gemeint ist hier Chuntaiji Galdan (Galdan Bošigt Hān), der Herrscher der Dzungaren (1676-1697); vgl. hierzu Grousset, René: Die Steppenvölker. Attila Dschingis Khan - Tamerlan. Essen 1975, pp. 708-714.

17 Dies = Tsewanrabtan (Erdeni Suruqtu Ba'atur Quntaiji [eigentlich: Sa-skyong sTags-rTsepa IHa-rGyal rab-brtan]), Herrscher der Dzungaren (1697-1727); vgl. hierzu Grousset (1975), pp. 715-719.

18 Auch - und vor allem - die im vorliegenden „Tractat“ gegebenen Angaben zur Geschichte der Kalmücken (und hier wiederum der inner-kalmückischen Auseinandersetzungen im 17. Jh.) dürfte einen wesentlichen Teil von v. Strahlenbergs „Bericht über eine Reise zu den westlichen Kalmücken“ ausgemacht haben. Angaben zu den Kalmücken - wenngleich weniger zu deren Geschichte, als vielmehr zu Sprache (hier in Gestalt eines „Vocabulariums“ [pp. 137-156]) - finden sich ja auch in nennenswertem Umfang im „Nord- und Ostlichen Theil von Europa und Asia“" (vgl. auch p. 46 f.). 
le Grand Contaisch a besoin de lever des trouppes, il assemble, en moins de rien ${ }^{20} / \mathrm{m}$ hommes, en ne prenant qu'un homme d'entre 10 . familles.

(7) Les maisons des Bouchars sont de pierre, \& passablement bonnes, leurs meubles sont en petit nombres \& de peu d'ornement. Ils n'ont ni tables ni chaises. L'on ne voit dans leurs chambres que quelques coffres Chinois ornez de fer, sur les ques ils ajustent, pendant le jour, les matelats dont ils se servent la nuit, et les couvrent d'un tapis de cotton bigaré. Ils y ont encore un rideau brodé à [8] fleurs, \& à figures, de differentes couleurs, \& une espece de bois de lit, haut de $1 / 2$. aune, \& large de 4 . aunes environ, qui leur sert de couche, \& qu'ils cachent le jour sous un tapis.

(8) Ils couchent nuds comme la main durant la nuit, mais dès qu'ils sont levez, on les voit toûjours habillez, \& assis à la Turque, ayant les jambes croisées sous eux.

(9) Ils se piquent de quelque propreté dans leur nourriture, Les esclaves qu'ils prennent ou achetent, chez les Calmuques, Russiens, \& autres peuples voisins, font la Cuisine dans la chambre du maitre. Pour cêt effet ils y ont selon la grandeur de la famille, plusieur marmites de fer, murées dans une espece de foyer, pres d'une cheminée, qui sert en même têms à chauffer la chambre en tems d'hiver, Il y en a qui ont aussi des petits fours, qui se font, comme le reste de leur murailles, de terre grasse ou de briques cruës.

[9] (10) Leur vaisselle consiste en quelques plats \& ecüelles de Capua (qui est une sorte de bois) ou de porcelaine, $\&$ en queques vases de cuivre, pour bouillir le thée, \& pour chauffer l'eau, dont ils ont besoin pour se laver, vne piece de toile bigarée de cotton leur tient lieu de nappe $\&$ de serviettes, Ils ne se servent ni de couteaux, ni de fourchettes. Les viandes leur êtant servies decoupées ils les prennent, \& achevent de les dechirer, avec les doigts, leur cuillieres sont de bois \& de la façon de celles, dont nous nous servons dans nos cuisines pour ecumer nos pots.

(11) Les mets, dont ils se nourrissent ordinairement, sont des hachis, dont ils remplissent souvent des especes de patez, de la Figure d'un croissant, lors qu'ils font de longs voiages, sur tout en hiver, ils se munissent d'une provision de ces patez, qu'ils portent auec eux dans un sac, apres les avoir exposés à la gelée \& ils en font d'assez [10] bonnes souppes, en les faissant recuire dans de l'eau bouillante.

(12) Leur boisson quotidienne est le thée. Ils en ont une espece noire, qu'ils appretent avec du lait, du sel \& du beurre \& le mangent 
avec du pain, lors qu'ils en ont, ou le boivent selon leur appetit.

(13) Leurs habillements, quant à ceux des hommes, ne different que fort peu de ceux des Tartares. Ils portent des habits longs jusqu'aux gras des jambes, ajant des manches larges vers l'epaule, \& étroite vers le poignet \& ils se ceignent d'echarpes, comme les Polonois.

Les habits des femmes ressemblent en tout â ceux des hommes, \& sont ordinairement piquez de cotton. Elles portent des pendants d'oreilles, de la longueur d'un $1 / 4$ d'aune \& qui descendent souvent jusques sur les epaules. Elles partagent leurs cheveux en plusieurs tresses, qu'elles entrelacent, \& allongent de rubans noirs, orodez d'or ou d'argent, \& de grandes [11] touffes de soye \& d'argent, qui leur pendent jusqu'aux talons.

Trois autres touffes moins grandes leur couvrent la gorge. Elles ont des colliers ornez de perles, de petites monnoyes, \& de plusieurs babioles argentées ou d'orées, \& luisantes. Tous generalement, hommes $\&$ femmes de quel age qu'ils soyent, portent sur eux, duns un \& ui de cuir fort mince, \& en guise de reliques, des prieres écrites à la main, que leurs pretres leur distribuënt, $\&$ dont ils ne font pas moins de cas que les Russiens de la Croix, \& des Saints.

Quelques femmes, \& sur tout les filles, ont les ongles teints de rouge. La couleur qu'elles y employent, \& qui y dure long tems, se tire d'une herbe, appellée dans la langue Bouchare, Kena; Elles la sechent, la pulverisent, la melent d'alun pilé, \& l'exposent durant 24. heures á l'air, avant que de s'en servir.

[12] Tant les hommes que les femmes portent des culottes \& des bottes de cuir de Russie fort legeres \& sans talon, pu des bas de cuir. Mais lors qu'ils sortent de la maison, les uns \& les autres se servent de galoches, ou de mules à hauts talons, comme les Turcs, \& les quittent en rentrant chez eux. L'un \& l'autre sex e se sert pareillement des mémes bonnets \& coëffures, á cela prés, que les femmes, \& sur tout les filles, parent les leurs de clinquants, de petites monnoyes, \& de perles Chinoises. La seule marque, à la quelle on peut distinguer les femmes d'avec les filles, c'est que les femmes portent sous les bonnets un linge long, lequel, apres luy avoir fait faire le tour du col, elles noüent par derriere, desorte qu'un bout de ce linge pend le long des reins.

(14) Le pays est tres abondant en toutes sortes de fruits \& en vins. La chaleur de l'été y est si excessive, qu'on á de la peine á la supporter hors des maisons. 
[13] (15) Les habitants ont ordinairement le teint bazane, \& la chevelure noire, bien qu'il y en ait aussi, qui sont fort blancs, beaux \& bien faits.

(16) Ils ne manquent pas de politesse, \& ils sont sur tout bien faisants envers les étrangers.

(17) Ils sont naturellement avides de gain, \& adonnez au trafic, ils en font beaucoup dans la Chine, en Perse, aux Indes, et en Russie. Ils sont tellement versez dans le negoce, que ceux qui commercent avec eux, á moins de s'y bien connoitre, ne manquent jamais d'étre surfaits ou duppez.

(18) Ils n'ont pas d'autre argent monnoyé que des Copeiks de cuivre, qui pesent un solotnik, C'est à dire, à peu pres le tiers d'une once.

Lors qu'ils ont de gros payements à recevoir, ou à faire, en or ou en argent, ils les reglent par la balance; Ils suivent en cela l'exemple des Chinois \& d'autres de leurs voisins.

[14] (19) Leur langue \& leur religion different \& bien des choses de celles des Turcs, \& des Persans, \& ressemblent neant moins aux unes \& aux autres.

Ils ont leur Alcoran, qui est le vieux Testament des Chretiens, mutilé $\&$ falsifié en bien des endroits,

Ils n'en attribüent pas la composition á Mahomed, mais à Dieu même, qui l'a communique aux Hommes, disent ils, par le moyen de Moyse \& des Prophetes, Mais ils sont persvadez que Mahomed en afait une explication, \& en a tiré une morale, qu'ils sont obligés de reconnoitre \& de suivre.

(20) Voici l'idée qu'ils se font de Jesus Christ: La Sainte Vierge, à leur dire, etant une pauvre orpheline, ses plus proches parents ne pûrent s'accorder, qui d'entre eux seroit chargé de son education, Pour terminer leur dispute, ils convinrent de la decider par le sort. Ils jetterent une plume dans un vase rempli d'eau, \& y tremperent tour á tour chacun un doigt. [15] La convention portoit, que celui, au doigt duquel la plume s'attacheroit de maniere qu'il pût la retirer de l'eau, seroit le pere nourricier de l'enfant; Tous y perdirent leur latin, à la reserve de Zacharie. La plume, quoy qu'elle fût allee au fond de l'eau, vint s'attacher à son doigt \& luy fit adjuger l'education.

Il s'en chargea avec plaisir, \& transporta 1'enfant dans sa maison. Mais un jour, qu'il etoit obligé de vaquer à quelque fonction, qu'il avoit dans le Temple, il oublia si bien d'avoir laissé l'orpheline toute seule, \& 
enfermée chez lui, qu'il resta 3. fois 24 . heures dehors, sans penser à elle. S'en etant enfin ressouvenû, \& craignant que la faim ne l'eût emportée, vû qu'aucun des autres parents n'en pouvoient avoir soin, la maison etant fermée à clef, il y courût au plus vite. Mais quelle fût sa surprise, quand, au lieu de trouver la pauvre enfant morte ou mourante, il la vit en bonne Santé \& entourée de toutes sortes [16] de bonne mangeaille; Son étonnement ne cessa qu'après que l'enfant lui eût appris, que Dieu les luy avoit envoyées.

Etant parvenüe à l'age de 14 . ans \& ayant été incommodée pour la premiere fois, comme toutes les femmes le sont ordinairement à cêt age là, cette sainte fille fût se baigner dans une fontaine, qui étoit dans une grande forêt. Lá elle entendit une voix, qui luy fit d'abord beaucoup de peur: Elle se hata de reprendre ses habits pour s'enfuir, mais, avant qu'elle put achever de les remettre, un ange, qui lui apparût, lui annonça qu'elle deviendroit enceinte d'un fils, qu'il lui commanda d'appeller Isay, lors qu'elle en seroit accouchée, Marie repliqua sagement, qu'elle auroit de la peine à accoucher, n'ayant jusques là jamais eu de commerce avec personne, qui eut pû l'engrossir. Mais l'ange, apres lui auoir soufflé sur la gorge, lui fit comprendre ce Mistere; \& 1'instruisit de tout ce qu'elle [17] avoit besoin de savoir. En effet elle devint grosse, des ce moment même, comme 1'ange l'avoit prédit. Le terme de sa delivrance s'approchant, Marie alla se cacher de honte dans la méme forêt, où l'ange lui etoit apparû, \& les douleurs l'y ayant surprise, elle s'appuya, pour se soulager, contre un tronc d'arbre toutsec, \& accoucha, dans cét estat, du fruit qu'elle portoit. Ce qu'il y eût de particulier, c'est que dans le méme instant le tronc commença à pousser des feuilles, \& toute la contrée d'à l'entour à germir \& à fleurir, \& que des anges survenûs prirent l'enfant qui, venoit de naitre, le baignerent dans une fontaine, qui se trouva tout a coup, à deux pas de lá, dans un endroit où il n'y en eût jamais auparavant, \& le rendirent ensuite à sa Mere.

Celle-ci, apres cette expedition retourna vers ses parents, qui la reçurent avec beaucoup d'imprecation \& de mauvais traitements. Elle les [18] essuya avec beaucoup de tranquillité, sans se donner la peine de s'excuser du crime, qu'on luy imputoit. Ellepria seulement son fils de plaider sa cause. Il le fit sur le champ, justifia entierement sa Mere, \& expliqua aux parents tout le mistere d'une naissance si peu naturelle, \& si miraculeuse. Dans la suite du têms, le Jeune Jsay devint un grand Prophete, \& un Docteur de beaucoup d'autorité. Mais il fût generalement 
haî \& persecuté de tout le monde, \& sur tout des plus grands hommes de son tems, des quels il essuya quantité de traverses \& de tribulations. Ils attenderent méme plusieürs fois, quoy que sans succés, à sa vie, \& ils detachement enfin deux personnages considerables, pour se defaire de lui à quelque prix que ce fût. Mais Dieu fit echoüer un si pernicieux dessein dans le moment même qu'ils comploient de l'executer. Il enleua tout à coup Isay de ce monde, \& le transporta tout en vie au Ciel. Qui plus est il punit les deux assassins d'une maniere singuliere [19] Il transforma successivement leur figures en celle d'Isai, \& les exposa par la à la fureur du peuple, qui, trompé par cette ressemblance les fit pitoyablement mourir.

(21) Bienque les Bouchars ne fassent aucun conte de la passion de JEsus Christ,, ni du mistere de nôtre redemtion, ils n'en croyent pas moins la resurrection, \& une autre vie: mais ils ne sauroient se persuader, que jamais mortel puisse être damné éternellement. Ils croyent, au contraire, que come les demons nous induissent au peché, ce sera aussi à eux à s'en charger, \& à en porter les peines.

(22) Ils croyent de plus, qu'au dernier jour du monde, tout ce qui existe, à la reserve de Dieu, sera aneanti, que par consequent, toute creature vivante, les anges, les Diables, \& Jesus Christ même, mouront, $\&$ qu'apres la resurrection, tous les hommes, excepté un petit nombre d'elûs, seront purifiez, c. a. d. chatiez par le feu, chacun à proportion de ses pechez dela grieveté [20] desquels Dieu connoitra, moyennant une balance; Ils croyent qu'il y aura 8. differents Paradis (qu'ils appellent array) pour les bons, 7. differents enfers Pour les mechants, que c'est dans ces enfers, que les pecheurs seront, plus ou moins purifiez par le feu de la punition, à proportion des pechez, qu'ils auront commis en ce monde; que les pecheurs les plus enormes, \& qui sentiront le plus vivement le feu de la punition, ce sont les menteurs, les fourbes, qui se plaisent à tromper, \& les boutefeux, qui sement de la desunion, \& des sujets de disputes, parmi leurs prochains; que ceux qui ne sentiront aucune atteinte du feu, c. a. d. les elûs, seront choisis parmi les bons; sçavoir de 100 . hommes un, \& de 1000 . femmes une, \& que ce petit troupeau sera transporté dans un des Paradis susdits, ou ils joüront de toutes sortes de felicité, jusqu'á ce qu'il plaira à Dieu de crêer un nouveau monde, au lieu de celui d'àpresent.

[21] (23) C'est uns peché, selon eux, que de dire, que Dieu est au Ciel. Dieu, disent ils, se trouve par tout; donc, c'est deroger à sa toute 
presence que de dire, qu'il se tient dans un endroit fixe.

(24) Ils ont tous les ans un jeune de 30. jours, à commencer du 15 . Juillet jusqu'à la mi. Aoust. pendant lequel têms ils ne goutent absolument de rien, tant qu'il fait jour, mais en échange, ils mangent deux fois la nuit; l'une, dès que le soleil est couché, \& 1'autre à minuit; il leur est defendû de se servir d'autre boisson durant ce jeune, que du thée. Quiconque contrevient en la monindre façon à ces ordonnances, est condamné sur Ie champ, à donner la liberté au meilleur de ses Esclaves, ou un repas à foixante personnes, $\&$ de souffrir outre cela, 85. coups, que le grand Prêtre, qu'ils appellent Aguns, luy fait apliquer sur le dos nud, moyennant une epaisse courroye de cuir, qu'ils nomment Dura. j'ay nean moins remarqué, que le petit peuple n'observe pas egalement la loy de ce jeune, [22] \& que sur tout, les travailleurs ont permission de manger en plein jour.

(25) Ils sont cing prieres par jour

1. avant l'aurore.

2. vers le midy.

3. aprez midy.

4. au soleil couchant \&

5. à la troisieme heure de la nuit, \& ce sont toujours leurs Abis, qui sont une espece de Pretres, qui en donnent le signal.

(26) Ceux qui sont assez savants pour savoir lire \& expliquer des livres, sont en grande consideration parmi ce peuple. Et on les apelle Mula qui veut dire un homme celebre \& de merites.

(27) Les femmes des Bouchars, lors qu'elles acouchent, sont reputées impures durant 40 . jours aprez leur delivrance, \& n'oseroient pas seulement prier Dieu tant que dure cette pretendüe impureté.

[23] (28) Au troisieme jour aprez la naissance d'un enfant, le Pere ou quelquun de plus proches parents, luy donne un nom \& luy fait en même téms present d'un bonnet, ou d'un linge, \& quelques fois d'un habit, si les finances le permettent.

La circoncision des jeunes garçons se fait par quiconque s'y entend, lors qu'ils sont parvenus à l'age de 7.8. ou de 9. ans, \& pour solemniser cette ceremonie, le Pere donne ordinairement un festin à la famille \& à ses amis.

(29) Les Bouchars, qui veulent se marier, sont obligés d'acheter leur femmes, comme nous achetons nos chevaux. Ils en payent plus ou moins à proportion qu'elles sont plus ou moins belles, de sorte que le moyen le 
plus sûr de s'enrichir c'est d'avoir quantité de filles à marier. Leurs mariages se font avec les ceremonies fuivantes. Il est defendu aux personnes qui veulent s'epouser, de se voir \& de se parler depuis le jour des fiançailles, [24] jusqu' à celui des nôces. Elles le celebrent pendant trois jours, qu'ils passent ordinairement, comme les trois grandes fêtes, qu'ils ont tous les ans, à bien manger \& boire. La veille des noces, quantité de jeunes filles vont s'assembler vers le soir chez la promise, $\&$ s'y divertissent jusqu' à minuit, à jouër, à danser \& à chanter. $\mathrm{Au}$ lendemain premier jour des noces, les conviez s'assemblent dez le matin chez la future \& 1'aident à tout preparer pour la ceremonie. Lorsque tout est prêt, on fait avertir le futur, qui arrive bientôt aprez, en compagnie de dix à douze de ses parens ou amis, \& suivi de quelques jouëurs de flûtes $\&$ d'un Abis, qui chante en battant deux petits timbales.

Desque le future est arrivé, il se tient une course de chevaux, aprés la quelle, on distribüe les prix aux Cavaliers les plus adroits les prix sont au nombre de 6. de 8. \& de 12. selon 1'opulence des nouveaux mariez, \& consistent ordinairement en Damas, en zibelines, en [25] renards, en kitaik, en toile de cotton, ou en autres effets pareils. Le même fête se donne aussi à la circoncision des enfants.

Les epoux ne se voyent point durant la copulation, ou ceremonie du marriage, \& ils repondent de loin aux questions, que le prêtre leur fait. Aprés la ceremonie, le future retourne chez lui dans le même ordre qu'il étoit venû, \& y regale ceux qui l'avoient accompagné.

L'Après dinée il revient avec le même cortege chez la future, \& obtient la permission de luy parler, aprez quoy il retourne de nouveau chez luy, \& de là, sur le soir, chez la future. Il la trouve alors couchée, \& il se met tout habillé, \& en presence de toutes les femmes invitées, mais pour un moment seulement, à son coté. La même comedie se jouë pendant trois jours consecutifs, \& ce n'est qu'au soir du troisieme, que le nouveau marié est en droit de coucher en effet $\&$ sans temoins avec sa promise. Il luy seroit [26] honteur d'y attenter plutòt. Enfin ce n'est qu' au $4^{\text {me }}$ jour qu'il amene sa semme chez luy.

Il y an a parmi ces maries, qui stipulent exprez, qu'il leur soit permis dé rester encore quélque tems, \& sou vent une année entiere, chez leurs parents, \& en ce cas la, les epoux y demeurent avec elles. Mais, s'il arrive que la femme vienne à deceder sans enfants pendant cet intervalle, ses parents heritent de tout ce que l'epoux luy avoit donné, à moins que ceux cy, au bout de l'année du deuil, ne soient assez genereux pour luy 
en restituër la moitié.

(30) La polygamie est regardée, à la verité, parmi les Bouchars, comme une espece de peché, mais elle n'est jamais punie, \& il y en a, qui ont impunement jusqu'à dix femmes \& au delà.

(31) Tout mary, qui n'est pes content de sa femme, est le maitre de la r'envoyer, en luy laissant emporter tout ce qu'il luy avoit donné durant [27] leur mariage. Et s'il arrive que ce soit la femme qui veuille se separer du mary, elle est pareillement la maitresse de se retirer, mais sans emporter la moindre chose de ce qui luy apartenoit.

(32) Lors qu'un Bouchar tombe malade, voicy le remede dont il se sert; Un Mula luy lit un passage de quelque livre, souffle à plusieurs reprises sur luy \& d'un couteau bien aiguisé fait plusieurs gesticulations par dessus \& à coté du visage du patient. Ils s'imaginent que par cette operation, ils coupent la racine de la maladie, qu'ils disent dailleurs être 1'ouvrage du Diable.

(33) Enfin s'il arrive, qu'un Bouchar vienne à mourir, un Ptêtre luy met l'Alcoran sur la poitrine, \& recite quêques prierez, aprez quoy on porte le mort à son tombeau, qu'ils choisissent ordinairement dans quelque bois agreable \& qu'ils entourent en suite d'une haye ou d'une espece de palissades.

[28] Chap. IV.

De La derniere Revolution arrivée dans la petite Boucharie. ${ }^{19}$ (1)

Bosto Cham, ou Bosugto-Cham ${ }^{20}$ Prince des Calmuques qui campoit ordinairement sur les bords d'un lac, appellé Jamisch, \& dans les desserts adjacents, faisoit elever à sa Cour trois neveux, fils de son frere.

19 Die hier im Folgenden beschriebenen Ereignisse stellen einige Aspekte der innerkalmückischen Auseinandersetzungen - oder vielmehr deren Wahrnehmung durch die westlichen Reisenden und russischen Autoritäten - dar, die sich nahezu durch das gesamte 17. Jh. zogen. Eine erste ausführliche Darstellung der Ereignisse findet sich bei P. S. Pallas, der einen Abriß der kalmückischen Geschichte - und vor allem Darstellungen der Genealogien der kalmückischen Fürsten - liefert (Pallas, Peter Simon: Sammlungen historischer Nachrichten ilber die Mongolischen Völkerschaften. Erster Theil. St. Petersburg 1776, pp. 24-96); eine moderne, wenn auch eher skizzenhafte, Darstellung der Ereignisse findet sich bei Grousset (1975), pp. 701-719.

20 Zu Chụtaiji Galdan (Galdan Bošigt Hुān) cf. oben Anm. 16. 
Ayant pris en aversion l'ainé de ces neuveux, il resolût de s'en defaire \& n'ayant pas de juste raison à alleguer contre Luy, il eut recours à un homme d'une force extraordinaire, qui sous pretexte de jouër \& de lutter avec le jeune Prince, le maltraita tellement, que peu de jours aprez il en mourût.

(2) Bosto Cham eut voulû faire passer cette mort pour un effet d'un mal heureux hazard. Mais on en devina [29] bien tôt la veritable cause. Entre autres Zigan-Araptan, ${ }^{21}$ frere puisné du defunt n'y fût pas trompé. Informé de la disgrace de son ainé, \& se croyant menacé du même sort que Luy, il jugea qu'il seroit de la prudence de s'eloigner du danger. Suivi de ses partisans, $\&$ de ses domestiques, il prit le parti de s'evader secretement.

(3) Bosto-Cham faché de la retraite de son neveu, mit tout en usage pour le faire revenir. Il ordonna à Danchinombu, frere cadet de ZiganAraptan, de l'aller chercher par tout le païs \& de tacher de le ramener.

(4) Danchinombu ne manqua pas de deligence. Il atteignit son frere au passage d'une riviere, \& ayant trouvé moyen de l'entretenir, il n'oublia rien pour le persuader de retourner vers leur oncle. Il luy representa, que leur ainé, par son caractere hautain \& remuänt, \& par sa mauvaise conduite, avoit causé luy même son malheur; que Bosto Cham forcé par le bien de l'Etat, n'avoit pû se dispenser [30] de le faire mourir; Mais qu'eux, etant exempts des defauts du defunt, n'avoient rien de pareil à aprehender. Enfin il l'exhorta, le pressa, \& le conjura de ne pas continuër sa fuite.

(5) Zigan-Araptan, outré de la mort de son frere, \& se defiant de la fin cerité d'un oncle si promt à depecher ses neveux fût sourd à toutes ses persuasions. Il dit pour toute reponse à son Cadet, qu'il pouvoit retourner seul chez. Bosto-Cham, \& y faire le parasite tant qu'il luy plairoit; mais que, quant à lui même, il etoit resolu de se passer desormais des bonnes graces d'un prince si denaturé, \& qu'il trouveroit moyen de vivre par tout ailleurs si non avec le même agrement, au moins en plus de sûreté. Aprés cette declaration, sans vouloir plus êcouter son frere, il remonta à cheval, \& le quitta.

(6) Quelque téms aprés ces evenements, Bosto-Cham s'etant brouillé avec Zain-Cham, ou Zuzi Cham, Prince des Mongales, Amulon

21 Zu Tsewayrabtan (Erdeni Suruqtu Ba'atur Quntaiji [eigentlich: Sa-skyong sTagsrTsepa IHa-rGyal rab-brtan]) cf. oben Anm. 17. 
Bogdo-Cham, [31] Empereur de la Chine, pour empêcher ces deux Princes, ses voisins, d'en venir aux armes, interposa son credit \& son autorité, pour tàcher d'assoupir leurs demelez. Pour cet effet, il les requit par un ambassadeur, nommé Averna Alcanaibu, de s'assembler dans un endroit sur la frontiere, \& de terminer leurs differents à l'amiable, sous la mediation du Dalay Lama.

(7) Le Dalay Lama est une espece de Pontife, autant respecté parmi les Calmuques \& Mongales, que le Pape l'est parmi les Chretiens. La vie de ce personnage est sujette à plusieurs circonstances fort singulieres. Il ne se montre en public, que quand il s'a git de se faire adorer. Lors qu'il s'ingere dans quelque affaire Politique, c'est le Deva (qui est une sorte de Plenipotentiaire) qui s'en mele sous ses ordres. Mais ce qu'il y a de plus particulier, c'est qu'on luy fournit jour nellement, pour sa subsistance, une once de farine de trempée avec du vinaigre [32] \& une tasse de Thée. C'est de cette pitance, que le Dalay Lama, malgré le haut rang qu'il tient, \& malgré le grand pouvoir qu'il a, est obligé de se contenter. Je reviens á mon sujet.

(8) La proposition de l'Empereur de la Chine fût acceptée par Le Prince des Calmuques \& par celuy des Mongales. Leurs etmbassadeurs \& le Deva, de la part du Dalay Lama, se rendirent au Lieu du Congrés. Mais leurs Conferences, malgré les soins du Mediateur, furent sans effet. Les Ambassadeurs des deux Princes au lieu d'entrer, en matiére, s'amuserent à se disputer la preseance.

(9) Celuy de Bosto-Cham soutint, qu'elle etoit düe à son maitre par deux raisons: l'une, parce qu'il descendoit en ligne directe de ZingisCham, Prince anciennement connu, \& fort renommé parmi les Tartares; l'autre, parce que la puissance des Calmuques surpassoit d'autant celle des Mongales, que les cheveux de la tête, dit il, [33] surpassent les poils des sourcils. Cette comparaison piqua L'Ambassadeur de Zain-Cham. Il repliqua fierement, qu'il ne falloit qu'un bon rasoir pour les egaliser, \& rompit le Congrès. Ces Ministres eussent epargné bien du sang \& bien des malheurs à leurs patries, si, au lieu de perdre le têms en des contestations si frivoles, ils se fussent appliqués à assoupir les differents de leurs maitres.

(10) L'empereur de la Chine, informé de cêt êclat, \& prevoyant que la guerre entre les deux Princes seroit inevitable, delibera long têms sur ce qu'il auroit à faire. D'un coté, il redoutoit les forces \& l'humeur intrepide \& entreprenante de Bosto-Cham, \& il eût êté bien aise de le 
voir humilié; de l'autre coté, il êtoit à craindre, qu'en le commettant avec les seuls Mongales, moins puissants que les Calmuques, il n'eut de l'avantage sur eux \& que le remede ne fût pis que le mal. Et supposé que la partie eut êté egale, il luy paroissoit toûjours dangereux [34] de voir le feu de la guerre s'allumer si près de ses Etats. Après bien des considerations il resolut enfin, d'eloigner le peril le plus qu'il pourroit de ses frontieres \& de remettre le reste au têms, \& à la providence.

(11) Il insinua, pour cet effet, á Zain-Cham, qu'il y auroit trop de risque pour Luy, d'attendre que Bosto-Cham vint l'attaquer en Mongalie; \& que le droit du jeu seroit, de le brusquer \& prevenir, en fondant le premier sur luy $\&$ en penetrant, le plus avant que faire se pourroit dans la Calmuquie. Il est sêur, que rien ne decontenance plus un ennemi, qui se croit le plus fort qu'une attaque soudaine, dans un têms oú il croit qu'on ne songe qu'à se defendre.

(12) L'empereur ayant appuyé ses insinuations par quantité de magnifiques presents, tant en or, qu'en argent, \& par les promesses, qu'il fit sous main à Zain-Cham, de l'assister, en cas de besoin, de toutes ses forces celui ci se laissa persuader. Il assembla [35] le plus de trouppes qu'il pût, \& malgré la rigueur de la saison, se jetta comme un torrent dans la Calmuquie.

Les commencements de cette entreprise furent des plus heureux. L'avant garde de Zain-Cham rencontra, \& battit à platte couture celle des Calmuques, \& Dorzizap, frere de Bosto-Cham, y perdit la vie.

(13) Bosto-Cham qvelque surpris par ce coup imprevû, n'en fût point effrayé. Il en recût la prémiere nouvelle dans un têms, qu'il êtoit à prendre du Thée. Le Courier qui l'apporta luy ayant annoncé la defaite $\&$ la mort de son frere, \& que les ennemis n'etoient plus guére eloignez de lui, il en fût d'abord troublé \& voulant se hâter de donner quelque ordre, il renversa la tasse qu'il tenoit, $\&$ s'echauda les mains.

Voila, dit il, en riant, aux assistants, voilà ce qu'on gagne par le trop de vivacité. Si j'avois ête [36] moins promt, je ne me serois pas brulé.

Aprés cette reflexion, rentrant dans son sens froid ordinaire, il pensa à ce qu'il auroit à faire, \& ne fût pas long têms à prendre sa resolution. La profondeur des neiges 1'empechant d'agir avec succés, Il se contenta de resserrer d'abord son armée, \& d'etre sur ses gardes, ne doutant pas que les Mongales, enhardis par leur victoire, \& ne connoissants pas le pays comme luy, ne lui donnassent bien tôt quelque prise sur eux. Les effets montrerent, qu'il ne s'etoit pas trompé. 
(14) Afin de derouter d'autant mieux les Mongales, qui continuoient d'avancer, Bosto-Cham fit semblant d'avoir peur. Il monte promptement à Cheval, \& publie par tout, qu'il va tout quitter, \& qu'on n'aura de ses nouvelles qu'au bout de quelques années.

(15) Le bruit de cette resolution s'etant repandu jusqu'au camp des Mongales, Zain-Cham double sa marche, [37] et, pour atteindre d'autant plus tôt le pretendu fuyard, il detache par differents chemins deux corps volants, l'un de $8 / \mathrm{m}$. \& l'autre de $3 / \mathrm{m}$. hommes. C'etoit à quoy BostoCham s'attendoit. Instruit de cette, demarche, il tourne tout à coup sur ces deuz detachements; les enveloppe, \& les taille en pieces.

(16) Il n'en demeura pas 1a. Il mena promtement son Armée contre celle de Zain-Cham, \& lui presenta la bataille. Cette resolution etonna d'autant plus les Mongales, qu'ils ne s'y attendoient pas. Une peur Panique les saisit. Ils prennent honteusement la fuite avant d'etre attaque. Bosto-Cham les ayant poursuivis \& rejoints, les charge, les met en desordre, \& en fait un carnage terrible.

(17) On peut juger du nombre des Mongales, qui furent tuëz à cette bataille, par la quantité d'oreilles, \& de treffes de cheveux, que BostoCham leur fit couper. Il en eût la charge de [38] neuf Chameaux qu'il envoya à sa residence, comme une marque assurée d'une entiére victoire.

(18) La joye qu'il en eût ne l'empêcha pas de poursuivre les Mongales, qui etoient echappés de cette boucherie. Il se mit à leurs trousses à la tête de ${ }^{30} / \mathrm{m}$. hommes, \& les mena toujours battant, jusqu'à la muraille de la Chine, derriere laquelle en fin Zain-Cham se retira.

(19) Les nouvelles de ces succès êtant parvenuës à la connoissance de L'Empereur de la Chine, ce sage Monarque rècommença à se donner beaucoup de mouvements pour reconcilier les deux Princes. Il n'oublia ni persuasions, ni largesses, pour porter Bosto-Cham, à poser les armes. Mais il est rare qu'un vainqueur sache user avec moderation de sa victoire.

(20) Trop avide de gloire \& de vangeance Bosto-Cham, bien loin d'accepter les riches dons, que L'Empereur [39] luy offroit, les lui renvoya, \& ferma l'oreille à toute proposition d'accommodement. II exigea du Monarque de la Chine, qu'il eût à lui livrer Zain-Cham \& tous ceux qui s'etoient refugiez avec luy dans ses Etats au de faut de quoy il luy annonçoit, à luy même, la guerre. Bosto-Cham se seroit epargné bien des malheurs, s'il eut êté plus traitable, \& moins audacieux. 
(21) Une declaration si hautaine ne pût guere manquer d'avoir les suites qu'elle eût. Amulon Bogdo-Cham la recût comme un defi dans les formes, \& ne differa plus de prendre les armes. D'abord il fit marcher successivement plusieurs Corps d'armée; mais ils ne firent rien qui vaille. Bosto-Cham fût assez heureux, pour les mettre tous en fuite, à mesure qu'ils venoient à luy. Les trouppes de ce Prince etoient si braves, oû celles de L'Empereur si mauvaises, qu'un jour 1000. Calmuques battirent $20 / \mathrm{m}$. Chinois, \& une autre fois $10 / \mathrm{m}$. enculbuterent $80 / \mathrm{m}$. [40] (22) La providence n'eleve souvent les mortels au faite du bonheur que pour leur faire mieux sentir leur chûte. Bosto-Cham en est un exemple. Amulon Bogdo-Cham, pour mettre fin aux progrés de son ennemi resolut de le combattre avec toutes ses forces, \& de l'accabler par le nombre. Il assembla $300 / \mathrm{m}$. hommes, bien pourvûs de tout ce qu'il falloit pour une vigoureuse guerre, \& un train d'artillerie de 300 . pieces de Canons.

(23) Cette formidable Armée, dix fois plus forte, que celle des Calmuques, environna de toute part leur camp. L'Empereur etoit presque seur de la victoire: Mais preferant toujours la voye de la douceur à la violence, il voulût bien offrir encore la paix à Bosto-Cham, avant de l'assaillér. Il luy la fit proposer, avec de conditions aussi honorables \& aussi avantageuses, qu'on eut dit, qu'il se fut luy même trouvé dans l'embarras, où l'autre se trouvoit. Bosto-Cham cependant enflé de ses prosperitez passées ne connût [41] pas, ou meprisa le peril, qui le menaçoit. Il rejetta toutes ces propositions avec dedain, \& força, pour ainsi dire, 1'Empereur à se servir de tous ses avantages. Les deux armées se livrerent enfin une bataille sanglante, Bosto-Cham la perdit, \& eut bien de la peine à se sauver, avec une poignée de fuyards, dans les montagnes voisines.

(24) Il fut d'autant plus sensible à ce malheur, qu'il se l'etoit attiré par sa faute. Mais ce qu'il'affligea le plus, ce fut la perte de Guny, ou Any, sa femme, qui fût tuée dans le deroute. L'Empereur ayant trouvé son Corps parmi les morts, lui fit arracher la tête \& l'emporta avec luy, pour en orner son triomphe.

Le malheur de Bosto-Cham ne se borna pas à cela; Manquant de vivres \& de fourage, dans les Montagnes, où il s'etoit retiré, la plus grande partie de son peu de monde, \& de ses chevaux, creva de faim \& de misere. Il fut trop heureux d'en echapper avec [42] une tres petite suite $\&$ de retourner ainsi presque seul, dans ses Etats. 
(25) Etant arrivé chex lui, il passa deux ans dans une mortelle affliction, exposé aux reproches \& aux plaintes de ses sujets, qui se ressentoient tous de sa defaite. Ne voyant nul autre moyen, pour se relever de son infortune, il voulût tenter d'ensortir par la voïe de la negociations, \& il se determina à envoyer Septenbaldius, son fils, au Dalay-Lama, à Berentola son intention êtoit apparemment, de recourir à 1'interposition de ce Pontife, \& de se remettre à son arbitrage, qu'il avoit ci devant refusé de reconnoitre. Mais Abay Dola Beck, Gouverneur de la ville de Camull, quoyque dependant de Bosto-Cham, fit arrêter Septenbaldius, qui passoit par son Gouvernement, $\&$ la petite suite qui l'accompagnoit; Il les envoya prisonniers à Pekin \& se soûmît luy même, avec tout son Gouvernement, à Amulon Bogdo-Cham.

(26) Ce fut un présent bien agreable á L'Empereur de la Chine. Il fit [43] trancher les têtes aux prisonniers, \& fit savoir au Gouverneur de Camull; qu'il le confirmoit dans son poste, \& qu'il luy promettoit sa grace, \& sa protection, à condition qu'il ne reconnût desormais d'autre maitre, que L'Empire de la Chine faute de quoy il le menaçoit des plus cruels supplices, \& de 1'exterminer lui \& toute sa race.

(27) La nouvelle de ce desastre mit le comble au des espoir de Bosto-Cham. Il convoqua tous ses sujets, les exhorta à vivre en paix, \& en bonne intelligençe entre eux, \& leur ayant donné la liberté de se retirer, chacun où il luy plairoit, il prit du poison, $\&$ mourût.

(28) Telle fût la fin de Bosto-Cham Prince d'un grand genie, \& de beaucoup de valeur.

Une suite d'heureux succès l'avoit rendû la terreur de tous ses voisins, \& l'avoit comblé de gloire. Un seul malheur le plongea dans le mepris, \& [44] dans le neant. Tant il est vrai, que les vicissitudes de la fortune sont de tout pays, \& que le vray moyen de se mettre à couvert de ses revers, c'est de se desier à têms de sa stabilité.

(29) Aprés le decés de Bosto-Cham, Zigan-Araptan, dont il a eté parlé ci dessùs, parût de nouveau sur le theatre Il s'etoit tenû caché, pendant la vie de son oncle; mais, des qu'il eut appris son trepas, il vint se presenter aux Calmuques, \& demanda à succeder au trone du defunct. Il en etoit effectivement le plus proche heritier; les Calmuques, dont il s'etoit acquis 1'affection dès son enfance, ne balancerent pas de lui prêter hommage. Les Bouchars, que Bosto-Cham avoit subjuguez quelque têms auparavant, suivirent cet exemple. D'autres Provinces, qui refusoient de s'y conformer, y fûrent obligées par les armes. 
(30) Zigan-Araptan ayant ainsi êté reconnû par tous les Etats de Bosto-Cham, les Bouchars le conduisirent [45] un jour vers un endroit particulier. C'êtoit un petit bois fort agreable par sa situation, \& qui ne consistoit qu'en 100. arbres touffûs \& d'une espece singuliere. Il s'y donna pendant quelques jours, plusieurs beller fêtes, aprés quoy l'on revetit solemnellement le nouveau Prince du titre de Contaisch, qui signifie un grand Monarque, \& l'on defendit sous peine de mort de l'appeller de son premier nom.

(31) Le nouveau Contisch meritoit bien cette distinction. C'est un Prince doüé de grands talents. Il a beaucoup de genie, de douceur, de courage \& de pieté. Il est actuellement en guerre avec 1'Empereur de la Chine, auquel il donne bien de la besogne.

(32) On raconte plusieurs particularités de sa vie. Je me contenterai d'en rapporter deux qui sont parvenües à ma connoissance. Le Contaisch se trouvant un jour à la chasse il arriva par accident, qu'un de ses [46] domestiques, lachant mal adroitement son arc, luy creva un oeil. Le reste de la suite, indigné de voir leur maitre borgne, se jetta sur le malheureux tireur, \& voulut le punir de mort. Mais le Contaisch s'y opposa. Laissés l'aller en paix, dit il à ses gens. Il ne faut juger d'un crime que par l'intention de celui, qui le commet. Celui cy m'a blessé sans dessein; sa mort ne me rendroit pas 1'oeil, qu'un hazard m'a fait perdre, Et non content de lui auoir sauvé la vie, il lui donna la liberté, àfin de le recompenser dit il, du danger qu'il auoit courû.

Un autre de ses sujets eût le malheur deperdre trois fois de suite tout son bien. Le Contaisch qui connoissoit d'ailleurs le merite de cet honnête homme, le remit chaque fois, avec beaucoup de generosité dans un êtat d'opulence: mais la fortune ne cessant pas de le perfecuter, \& l'ayant replongé dans une quatriéme mendicité, il implora de nouveau la munificence [47] du Contaisch; Surquoy ce Prince lui repondit en ces termes: Il te souvient, mon fils, que je t'ai assisté trois fois. Je le ferois encore celleci, si je ne jugeois par l'opiniatreté de ton mauvais sort que le Ciel semble t'avoir destiné à la pauvreté. Je n'oserois plus aider un homme, que Dieu lui - même abandonne si visiblement. FIN. 
